\title{
Weyl nodes with higher-order topology in an optically driven nodal-line semimetal
}

\author{
Xiu-Li Du, ${ }^{1, *}$ Rui Chen, ${ }^{2, *}$ Rui Wang, ${ }^{3,4,5, \dagger}$ and Dong-Hui $\mathrm{Xu}^{1, \ddagger}$ \\ ${ }^{1}$ Department of Physics, Hubei University, Wuhan 430062, China \\ ${ }^{2}$ Shenzhen Institute for Quantum Science and Engineering and Department of Physics, \\ Southern University of Science and Technology (SUSTech), Shenzhen 518055, China \\ ${ }^{3}$ Institute for Structure and Function and Department of Physics, Chongqing University, Chongqing 400044, China \\ ${ }^{4}$ Chongqing Key Laboratory for Strongly Coupled Physics, Chongqing 400044, China \\ ${ }^{5}$ Center for Quantum Materials and Devices, Chongqing University, Chongqing 400044, China
}

(Dated: January 25, 2022)

\begin{abstract}
Creating and manipulating topological states is a key goal of condensed matter physics. Periodic driving offers a powerful method to manipulate electronic states, and even to create topological states in solids. Here, we investigate the tunable Floquet states in a periodically driven higher-order nodal line semimetal with both spatial inversion and time-reversal symmetries. We found that the Floquet Weyl semimetal states, which support both one-dimensional hinge Fermi arc and two-dimensional surface Fermi arc states, can be induced in the higherorder nodal-line semimetal by shining circularly polarized light. Moreover, we show that the location of Weyl nodes and the curvature of surface Fermi arcs can be tuned by adjusting the propagation direction and incident angle of light.
\end{abstract}

Introduction.-Recent developments of topological insulators and topological superconductors have attracted intensive interest in topological phases of matter. Creation and manipulation of topological states has become the central focus of condensed matter physics. Periodic driving through light fields opens a route towards engineering exotic Floquet topological states with highly tunability in solids via symmetry breaking or the modification of Dirac mass [1,2]. The last decade has witnessed great progress made in the exploration of various Floquet topological states in optically driven systems [3-30]. For instance, circularly polarized light (CPL) has been predicted to gap out the Dirac cone and create the quantum anomalous Hall insulator in graphene by breaking time-reversal symmetry (TRS) [3-10], while linearly polarized light can drive a band inversion and realize the Floquet topological insulator in semiconductor quantum wells [11]. In a recent experiment, the light-induced anomalous Hall effect in graphene driven by a femtosecond pulse of CPL has been experimentally observed [31]. Moreover, it has been reported experimentally that CPL can gap out the helical Dirac cones of three-dimensional (3D) topological insulators and produce Floquet-Bloch states [32, 33], which indicates the Floquet quantum anomalous Hall effect occurred in topological insulators. Apart from gapped Floquet topological phases, it has shown that Floquet Weyl semimetals (WSMs) can be generated in 3D topological insulators, Dirac semimetals, and nodal-line semimetals (NLSMs) by light driving [19-26].

Recently, higher-order topological insulators [34-39] with a brand-new bulk-boundary correspondence have become an exciting avenue in the study of topological phases of matter. Compared with conventional first-order topological insulators, the dimensionality of topologically gapless boundary states in higher-order topological insulators is more than one dimension below that of the bulk. Subsequently, the notion

\footnotetext{
* X.-L. D. and R. C. contributed equally to this work.

† rcwang@cqu.edu.cn

$\ddagger$ donghuixu@hubu.edu.cn
}

of higher-order topology was extended to gapless semimetallic systems [40-50]. Higher-order topological semimetals with point or line nodes exhibit unique boundary states absent in conventional first-order topological semimetals, such as 1D gapless hinge Fermi arc states connect the projections of bulk nodes on the hinges [42]. Moreover, the exploration of Floquet states with higher-order topology in periodically driven systems has attracted great interest recently [51-66]. So far, most attempts have focused on engineering a specific kind of Floquet higher-order topological state in periodically driven systems, but the investigation of light-driven systems with multiple higher-order topological states has been largely unexplored. Now, a natural question arises: Could we change the shape of bulk nodes with higher-order topology, such as from line nodes to point nodes, by an external periodic driving, such as CPL?

In this work, we study emergent Floquet topological states in a higher-order NLSM with both spatial inversion and timereversal symmetries under CPL illumination. Such a higherorder NLSM has two nodal rings in its bulk, and supports boundary states, including 1D hinge Fermi arc states and 2D drumhead surface states. Remarkably, when shining CPL propagates along the $z$ axis, each nodal ring evolves into a pair of Weyl nodes located along the $k_{z}$ axis, and a Floquet higherorder WSM state emerges due to TRS breaking. The higherorder topology of the parent NLSM is inherited by the Floquet WSM state, resulting in 1D hinge Fermi arcs connecting the projections of two Weyl nodes from different pairs. Moreover, the Floquet WSM state hosts 2D surface Fermi arc states as well. When the propagation direction of light changes to the $x$ axis or the $y$ axis, a Floquet WSM state is also formed. In this case, the location of Weyl nodes deviates from the $k_{z}$ axis accordingly. Importantly, the location of Weyl nodes can change with tuning the incident angle of CPL, implying that we can control Weyl nodes by adjusting the propagation direction and incident angle of CPL.

Model and method.-To study the periodically driven higher-order NLSMs by CPL, we focus on a class of higherorder NLSMs with both spatial inversion and time-reversal 
symmetries [45, 46, 49, 67, 68]. Particularly, we consider a spinless four-band tight-binding model on the 3D cubic lattice, which can be treated as a stack of alternating layers. The Hamiltonian in momentum space reads [46]

$$
\begin{aligned}
H(\mathbf{k}) & =t \sin k_{x} \Gamma_{1}+t \sin k_{y} \Gamma_{2} \\
& +\left[M-t\left(\cos k_{x}+\cos k_{y}+\cos k_{z}\right)\right] \Gamma_{3}+H_{\text {mass }},
\end{aligned}
$$

where the Dirac matrices are defined as $\Gamma_{1}=\sigma_{0} \otimes \rho_{3}, \Gamma_{2}=$ $\sigma_{2} \otimes \rho_{2}, \Gamma_{3}=\sigma_{0} \otimes \rho_{1}, \Gamma_{4}=\sigma_{1} \otimes \rho_{2}$, with $\sigma_{\mu}$ and $\rho_{\mu}(\mu=$ $1,2,3)$ two sets of Pauli matrices and $\sigma_{0}$ the identity matrix. $\rho$ acts on the subspace formed by the two alternating layers, and $\sigma$ means the sublattice degrees of freedom of each layer. $t$ describes the hopping amplitude, and $M$ is the Dirac mass that controls the first-order topology. For the sake of simplicity, we set $t=1$ in the following calculations. The fourth term $H_{\text {mass }}=i m\left(\Gamma_{1} \Gamma_{4}+\Gamma_{2} \Gamma_{4}\right)$ is represented as a real matrix, standing for an additional mass that is responsible to generate an NLSM with higher-order topology.

The inversion symmetry operator is $I=\Gamma_{3}$, and TRS operator is $T=\Gamma_{3} \mathcal{K}$ with $\mathcal{K}$ being the complex conjugate. In addition, the Hamiltonian also has chiral symmetry $C=\Gamma_{4}$ and mirror symmetry $M_{x \bar{y}}=\Gamma_{1} \Gamma_{4}+\Gamma_{2} \Gamma_{4}$ under which the Bloch Hamiltonian obeys: $M_{x \bar{y}} H(\mathbf{k}) M_{x \bar{y}}^{-1}=H\left(-k_{y},-k_{x}, k_{z}\right)$.

In the absence of $H_{\text {mass }}$, the Hamiltonian describes a 3D first-order Dirac semimetal, which has two Dirac nodes residing at $k_{z}= \pm k_{z}^{0}= \pm \arccos (M-2)$. The low-energy effective Hamiltonians around the Dirac points are given by

$$
H_{\mathrm{DSM}}(\mathbf{q})=q_{x} \Gamma_{1}+q_{y} \Gamma_{2} \pm \sqrt{(3-M)(M-1)} q_{z} \Gamma_{3},
$$

where $\mathbf{q}$ is momentum measured relative to the Dirac points. After turning $H_{\text {mass }}$ on, the Dirac semimetal evolves into a higher-order NLSM with two nodal rings on the mirror invariant plane.

Before studying the Floquet states induced by periodic driving, it is helpful to discuss the band structure of the undriven higher-order NLSM described by Eq. 1. Figures 1(a) and 1(b) show the two bulk nodal rings of the NLSM lying on the mirror plane $k_{x}=-k_{y}$ plane of 3D Brillouin zone and the band structure around them. Figure 1(c) plots the energy spectrum along the $k_{z}$ direction for the system with open boundaries in both the $x$ and $y$ directions. A pair of hinge Fermi arc states with zeroenergy flat bands (marked in red) terminate on the projection of nodal rings, which are the hallmark feature of higher-order topology in NLSMs. As shown in Fig 1(e), the hinge Fermi arc states are located on two mirror-symmetric off-diagonal hinges. Meanwhile, this kind of higher-order NLSM also supports flatband drumhead surface states in the region enclosed by the projections of nodal rings onto the surface Brillouin zone, as displayed in Fig. 1(f). With open boundary in the $x$ direction, the spectrum along the $k_{z}$ direction for $k_{y}=0$ is depicted in Fig. 1(d). The eigenvalues of drumhead surface states (marked in green) are pinned to zero energy due to chiral symmetry.

The circularly polarized driving field can be expressed as $\mathbf{E}(\tau)=-\partial_{\tau} \mathbf{A}(\tau)$, where $\mathbf{A}(\tau)=\mathbf{A}(\tau+T)$ is a timeperiodic, spatially homogeneous vector potential of period $T=2 \pi / \omega$, and $\omega$ is the frequency of light. The coupling
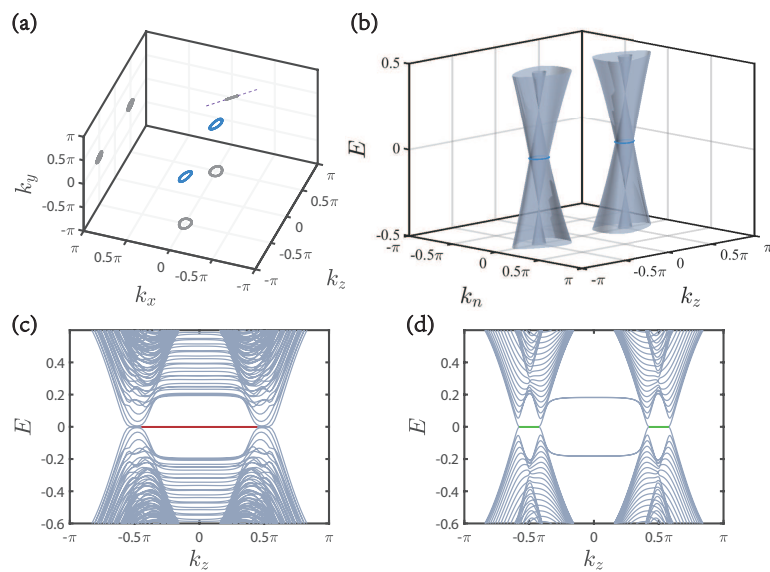

(d)
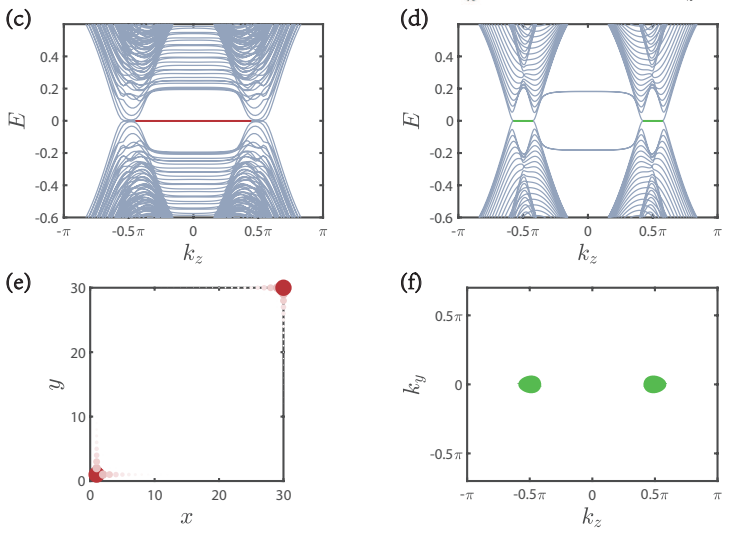

FIG. 1. Electronic structure of the undriven NLSM obtained by the tight-binding model described by Eq. 1. (a) Two higher-order nodal rings (blue) in the 3D Brillouin zone. The gray rings are the projections of the nodal rings on $k_{x}-k_{y}, k_{x}-k_{z}$ and $k_{y}-k_{z}$ planes, respectively. (b) The bulk energy spectrum as functions on the $k_{\mathbf{n}}-k_{z}$ plane with $\mathbf{k}_{\mathbf{n}}$ along the $k_{x}=-k_{y}$ direction. (c) The energy spectrum as a function of $k_{z}$ with the open boundary conditions along both the $x$ and $y$ directions. The red solid lines represent the hinge Fermi arc states. (d) The energy spectrum as a function of $k_{z}$ with the open boundary condition along the $x$ direction for $k_{y}=0$. The green lines represent the drumhead surface states. (e) The probability distribution of the hinge Fermi arc states of $k_{z}=0$. (f) The spectral density, calculated by the surface Green's function method, in the surface Brillouin zone defined on $k_{y}-k_{z}$ plane for $E=0$. The drumhead surface states appear in the regions bounded by the two projected nodal rings. The mass parameters are set as $M=2, m=0.2$.

to driving field $\mathbf{E}(\tau)$ enables electrons to acquire a phase factor as they hop between different lattice sites. Specifically, the phase factor can be obtained by the Peierls substitution: $t \rightarrow$ $t \exp \left[-i \int_{\mathbf{r}_{j}}^{\mathbf{r}_{k}} \mathbf{A}(\tau) \cdot d \mathbf{r}\right]$, where $\mathbf{r}_{j}$ is the coordinate of lattice site $j$. In this paper, we use the natural units $e=\hbar=c=1$. In the presence of $E(\tau)$, the Hamiltonian of the driven NLSMs becomes periodic and fulfills $H(\tau)=H(\tau+T)$.

According to the Floquet theory [69, 70], there exits a set of solutions of the time-dependent Schrödinger equation $|\Psi(\tau)\rangle=e^{-i \epsilon \tau}|\Phi(\tau)\rangle$, where $\epsilon$ is the Floquet quasienergy and the time-periodic function $|\Phi(\tau)\rangle=|\Phi(\tau+T)\rangle$ is called the Floquet state. Using the Fourier transformation $H(\tau)=$ $\sum_{n} e^{-i n \omega \tau} H_{n}$ and $|\Phi(\tau)\rangle=\sum_{n} e^{-i n \omega \tau}\left|\Phi^{n}\right\rangle$, the timedependent Schrödinger equation is mapped to a static eigenvalues problem

$$
\sum_{m}\left(H_{n-m}-m \omega \delta_{m n}\right)\left|\Phi_{\alpha}^{m}\right\rangle=\epsilon_{\alpha}\left|\Phi_{\alpha}^{m}\right\rangle,
$$

in the extended Floquet (or Sambe) space. Throughout, we focus on the situation where electron transitions through the 
absorption or emission of photons are very unlikely. This situation occurs for high frequency light. We can arrive at an effective Hamiltonian in the high frequency limit [71, 72]

$$
H_{\mathrm{eff}}=H_{0}+\sum_{n \neq 0} \frac{\left[H_{-n}, H_{n}\right]}{2 n \omega}+\mathcal{O}\left(\omega^{-2}\right) .
$$

In numerical calculations, we can choose the proper maximum value of $n$ by checking whether the results are converged.

Light propagating along the $z$ direction. - First, we introduce the CPL along the $z$-axis $\mathbf{A}=A(\eta \sin \omega \tau, \cos \omega \tau, 0)$ with $\eta= \pm$ labeling the handedness. Substituting Eq. 1 into Eq. 4, we get an effective Hamiltonian of the driven higherorder NLSM in the high frequency limit as follows

$$
\begin{aligned}
H_{\mathrm{eff}}(\mathbf{k}) & =t \mathcal{J}_{0}(A) \sin k_{x} \Gamma_{1}+t \mathcal{J}_{0}(A) \sin k_{y} \Gamma_{2}+H_{\text {mass }} \\
& +\left(M-t \mathcal{J}_{0}(A)\left(\cos k_{x}+\cos k_{y}\right)-t \cos k_{z}\right) \Gamma_{3} \\
& +\sum_{n \in \text { odd }, n>0} \frac{2 i \eta t^{2} \mathcal{J}_{n}^{2}(A)}{n \omega} \sin \frac{n \pi}{2}\left(\cos k_{x} \cos k_{y}\left[\Gamma_{1}, \Gamma_{2}\right]\right. \\
& \left.+\cos k_{x} \sin k_{y}\left[\Gamma_{1}, \Gamma_{3}\right]+\sin k_{x} \cos k_{y}\left[\Gamma_{3}, \Gamma_{2}\right]\right)
\end{aligned}
$$

where $\mathcal{J}_{n}(A)$ is the $n$-th Bessel function of the first kind. Comparing the effective Hamiltonian with its undriven case, we can see that the CPL not only renormalizes the original hoppings along the $x$ and $y$ directions, but also generates correction terms breaking TRS and chiral symmetry. However, the product of chiral symmetry and TRS $C T$ as well as mirror symmetry $M_{x \bar{y}}$ are still preserved.

Figure 2 shows the band structure obtained by the effective Hamiltonian Eq. 5. Interestingly, each bulk nodal ring evolves into a pair of Weyl nodes residing along the mirror invariant line $k_{x}=k_{y}=0$, depicted in Figs. 2(a) and 2(b). The Weyl nodes inhibit the higher-order topology from the nodal rings, resulting in a Floquet higher-order WSM state. As is evident in Figs. 2(c) and 2(e), the Floquet higher-order WSM state hosts 1D hinge Fermi arcs with flat bands connecting by the projection of two middle Weyl nodes from two different pairs. For each slice with a given $k_{z}$ within the two middle Weyl points, the effective Hamiltonian Eq. 5 reduces to a 2D second-order topological insulator with mirror invariant off-diagonal corner states. Moreover, such a light-induced higher-order WSM state also exhibits 2D surface Fermi arc states with zero energy dispersionless flat bands between a pair of projected Weyl nodes [see Fig. 2(d)] due to the existence of spectral symmetry $C T$ and mirror symmetry $M_{x \bar{y}}$. Accordingly, the surface Fermi arcs lose their curvature and form straight line segments [see Fig. 2(f)] which end at the projections of pairs of bulk Weyl nodes along the $k_{y}=0$ line in the surface Brillouin zone of the $k_{y}-k_{z}$ plane.

To better understand the Floquet WSM sates, we appeal to the low-energy continuum model of the driven NLSM within the framework of Floquet theory. The periodic driving field is introduced in the Hamiltonian by using the minimal coupling substitution $\mathbf{q} \rightarrow \mathbf{q}-\mathbf{A}(\tau)$. In the high frequency regime, the effective Floquet Hamiltonian up to the leading order is expressed as [2] $H_{\mathrm{F}}=H_{0}+\frac{\left[H_{-1}, H_{1}\right]}{\omega}$. Substituting the continuum model $H_{\mathrm{c}}(\mathbf{q})=H_{\mathrm{DSM}}(\mathbf{q}) \stackrel{\omega}{+} H_{\text {mass }}$ into $H_{F}$, we obtain
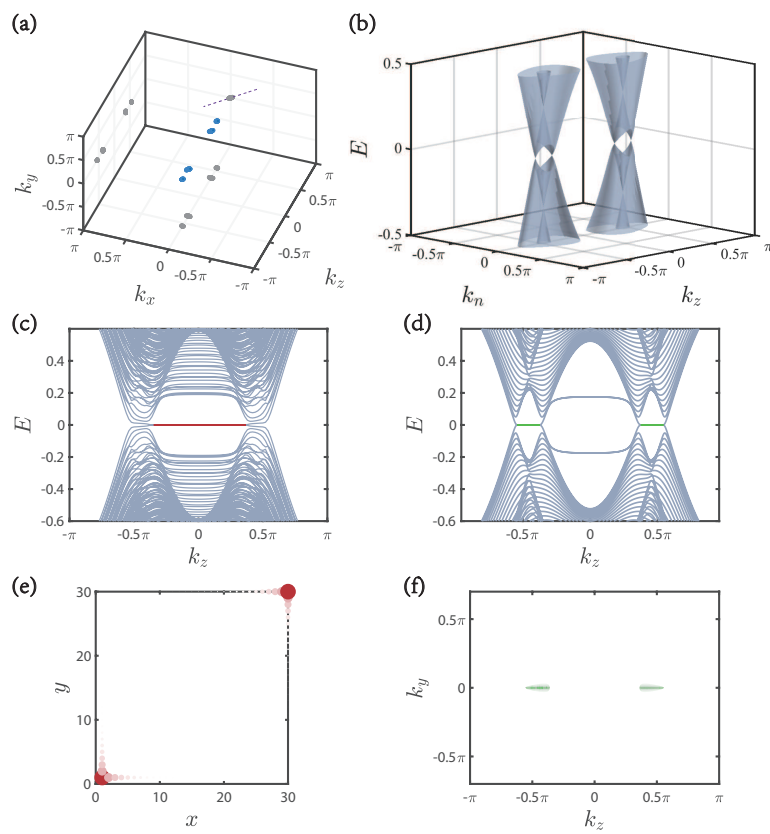

FIG. 2. Higher-order NLSM under CPL propagates along the $z$ axis. (a) The light-induced Weyl points (blue dots) in the 3D Brillouin zone. The gray dots are the projections of the Weyl points on $k_{x}-k_{y}$, $k_{x}-k_{z}$ and $k_{y}-k_{z}$ planes, respectively. (b) The bulk energy spectrum as functions on the $k_{\mathbf{n}}-k_{z}$ plane with $\mathbf{k}_{\mathbf{n}}$ along the $k_{x}=-k_{y}$ direction. (c) The energy spectrum as a function of $k_{z}$ with the open boundary conditions along the $x$ and $y$ directions. The red solid lines represent the hinge Fermi arc states. (d) The energy spectrum as a function of $k_{z}$ with the open boundary condition along the $x$ direction and $k_{y}=0$. The Green lines are surface Fermi arc states. (e) The probability distribution of the hinge Fermi arc states at $k_{z}=0$. (f) The location of surface Fermi arc states terminated at the projection of Weyl points on the surface Brillouin zone defined on the $k_{y}-k_{z}$ plane. The two line segments marked by green lines show the surface spectral density for $E=0$, which is obtained by the surface Green's function method. We choose parameters as $M=2$, $m=0.2, A=0.5$ and $\omega=3$.

the correction induced by CPL: $\Delta H=-\frac{i \eta A^{2}}{2 \omega}\left[\Gamma_{1}, \Gamma_{2}\right] . \Delta H$ is independent of $\mathbf{q}$ as $H_{\mathrm{DSM}}(\mathbf{q})$ is only expanded to linear order in $\mathbf{q}$. The correction $\Delta H$ enables each nodal ring to evolve into a pair of Weyl nodes located at

$$
q_{x}^{0}=q_{y}^{0}=0, \quad q_{z}^{0}= \pm \sqrt{\frac{\eta^{2} A^{4}+2 \omega^{2} m^{2}}{\omega^{2}(3-M)(M-1)}}
$$

This result is consistent with that obtained by using the tightbinding lattice model.

Light propagating along the $x$ direction.-To see how the propagation direction of CPL affects the properties of Floquet states, we consider CPL along the $x$ axis $\mathbf{A}=$ $A(0, \eta \sin \omega \tau, \cos \omega \tau)$. In this case, the effective tight-binding 


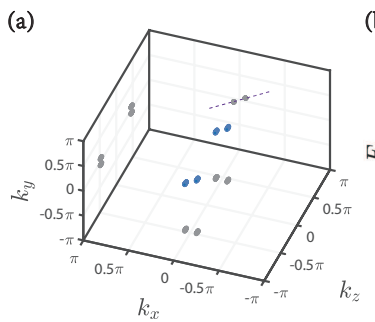

(e)

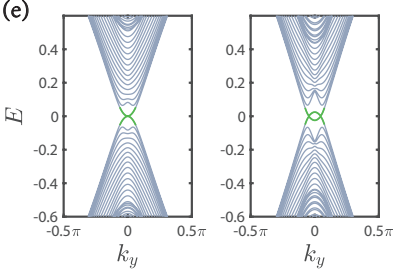

(b)

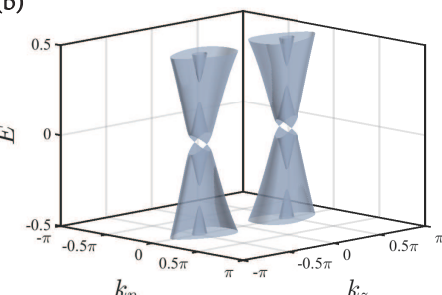

(f)

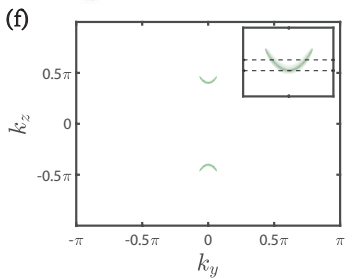

(c)

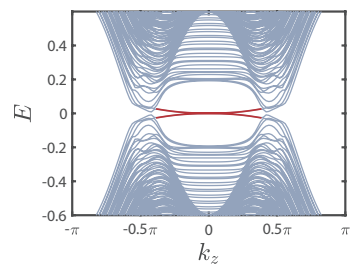

(g)

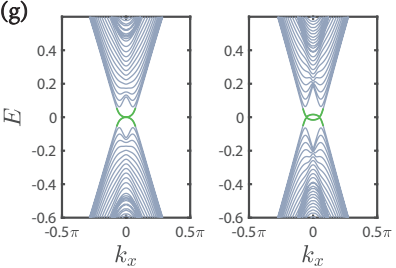

(d)

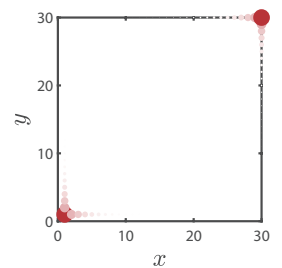

(h)

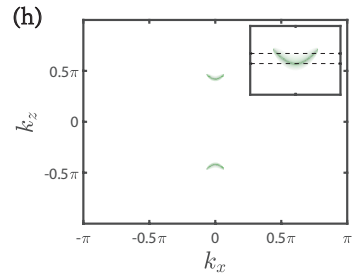

FIG. 3. Band structure of the driven higher-order NLSM under CPL propagates along the $x$-axis. (a) The Floquet Weyl points (blue dots) in the 3D Brillouin zone. The gray dots are the projections of the two pairs of Weyl points on $k_{x}-k_{y}, k_{x}-k_{z}$ and $k_{y}-k_{z}$ planes, respectively. (b) The bulk energy spectrum around the $k_{\mathbf{n}}-k_{z}$ plane on which the Weyl nodes are located. Here $\mathbf{k}_{\mathbf{n}}$ is along $k_{x}=-0.991 k_{y}$ direction, slightly deviating from the mirror plane. (c) The energy spectrum as a function of $k_{z}$ with the open boundary conditions along the $x$ and $y$ directions. The red solid lines mark the off-diagonal hinge Fermi arc states. (d) The probability distribution of the hinge Fermi arc states at $k_{z}=0$. (e) The energy spectra along the dashed line cuts shown in the inset of (f) for the open boundary condition along the $x$ direction. The surface Fermi arc states are marked by green color. (f) The location of surface Fermi arc states terminated at the projection of Weyl points on the surface Brillouin zone defined on the $k_{y}-k_{z}$ plane. The two curves marked in green show the surface spectral density for $E=0$, which is obtained by the surface Green's function method. The inset shows a zoomed-in view of the up Fermi arc. (g) The energy spectra along the dashed line cuts shown in the inset of $(\mathrm{h})$ for the open boundary condition along the $y$-direction. The surface Fermi arc states are marked by green color. (h) The location of surface Fermi arcs on the $k_{x}-k_{z}$ plane. We choose parameters the same as that used in Fig. 2.

Hamiltonian in the high frequency limit becomes

$$
\begin{aligned}
H_{\mathrm{eff}}(\mathbf{k}) & =t \sin k_{x} \Gamma_{1}+t \mathcal{J}_{0}(A) \sin k_{y} \Gamma_{2}+H_{\text {mass }} \\
& +\left(M-t \cos k_{x}-t \mathcal{J}_{0}(A)\left(\cos k_{y}+\cos k_{z}\right)\right) \Gamma_{3} \\
& -\sum_{n \in \text { odd }, n>0} \frac{2 i \eta t^{2} \mathcal{J}_{n}^{2}(A)}{n \omega} \sin \frac{n \pi}{2} \cos k_{y} \sin k_{z}\left[\Gamma_{3}, \Gamma_{2}\right] .
\end{aligned}
$$

The CPL along the $x$ axis renormalizes the hoppings in the $y$ and $z$ axes, meanwhile it also imposes a correction that breaks TRS and chiral symmetry to the undriven Hamiltonian. Besides, mirror symmetry $M_{x \bar{y}}$ is also broken by the CPL in the $x$ direction. By diagonalizing the effective Hamiltonian, we plot the energy spectrum in Fig. 3. Figures 3(a) and 3(b) show the location of two pairs of Weyl nodes in the 3D Brillouin zone and the bulk Weyl cone structure around the Weyl nodes, respectively. We found that the locations of two pairs of Weyl nodes deviate from the mirror plane $k_{x}=-k_{y}$ owing to mirror symmetry breaking. Again, the light-induced WSM state displays 1D off-diagonal hinge Fermi arcs, as shown in Figs. 3(c) and 3(d), indicating the unconventional bulk-boundary correspondence originated from higher-order topology. Yet the hinge Fermi arc states exhibit dispersive bands in the current case. In addition, the Floquet WSM supports curved surface Fermi arcs [see Figs. 3(f) and 3(h)], which is different from the case of Floquet WSM induced by the CPL along the $z$ axis where the surface Fermi arcs states show straight line segments. To better understand the formation of surface Fermi arcs, we show the spectra of surface Fermi arc states in Figs. 3(e) and 3(g).

Similarly, on the basis of the continuum model, we obtain the correction $\Delta H=\frac{i \eta A^{2} \sqrt{(3-M)(M-1)}}{2 \omega}\left[\Gamma_{3}, \Gamma_{2}\right]$. Thereby, the locations of Weyl nodes are at $\mathbf{q}=\left(q_{x}^{0}, q_{y}^{0}, 0\right)$ with

$$
\begin{aligned}
& q_{x}^{0}= \pm\left(4 m^{4}+B^{2}\right)^{\frac{1}{4}} \cos \left[\frac{1}{2} \arg \left(-2 i m^{2}-B\right)\right], \\
& q_{y}^{0}= \pm\left(4 m^{4}+B^{2}\right)^{\frac{1}{4}} \sin \left[\frac{1}{2} \arg \left(-2 i m^{2}-B\right)\right],
\end{aligned}
$$

where $B=A^{4}\left(3-4 M+M^{2}\right) / \omega^{2}$.

Light propagating along the $y$ direction.-Here, we consider the CPL along the $y$ axis: $\mathbf{A}=A(\cos \omega \tau, 0, \eta \sin \omega \tau)$. Correspondingly, we have the following effective Hamiltonian in the high frequency limit

$$
\begin{aligned}
H_{\mathrm{eff}}(\mathbf{k}) & =t \mathcal{J}_{0}(A) \sin k_{x} \Gamma_{1}+t \sin k_{y} \Gamma_{2}+H_{\text {mass }} \\
& +\left(M-t \cos k_{y}-t \mathcal{J}_{0}(A)\left(\cos k_{x}+\cos k_{z}\right)\right) \Gamma_{3} \\
& -\sum_{n \in \text { odd }, n>0} \frac{2 i \eta t^{2} \mathcal{J}_{n}^{2}(A)}{n \omega} \sin \frac{n \pi}{2} \cos k_{x} \sin k_{z}\left[\Gamma_{1}, \Gamma_{3}\right] .
\end{aligned}
$$

Although this effective Hamiltonian looks different from that in Eq. 7 for the CPL along the $x$ axis, the location of Weyl nodes and boundary states are quite similar to that are exhibited in Fig. 3. The correction term obtained by the continuum model is $\Delta H=\frac{i \eta A^{2} \sqrt{(3-M)(M-1)}}{2 \omega}\left[\Gamma_{1}, \Gamma_{3}\right]$.

Besides, we found that the higher-order NLSM is stable when CPL is incident normally on the mirror plane containing the two nodal rings.

Light propagating on the $x$-z plane.-Furthermore, we can rotate the propagation direction of CPL to the $x$ $z$ plane, the vector potential can be expressed as $\mathbf{A}=$ 
$\left(A_{x} \eta \sin (\omega \tau), A_{y} \cos (\omega \tau), A_{z} \eta \sin (\omega \tau)\right)$, where $A_{x}=$ $A \cos (\psi), A_{y}=A, A_{z}=A \sin (\psi)$ with $\psi$ the incident angle off the $z$ axis. When $\psi=0$ and $\psi=\pi / 2$, it reduces to the case of CPL along the $z$ axis and the negative $x$ axis, respectively. The effective Floquet-Bloch Hamiltonian is given by

$$
\begin{aligned}
& H_{\text {eff }}(\mathbf{k})=t \mathcal{J}_{0}\left(A_{x}\right) \sin k_{x} \Gamma_{1}+t \mathcal{J}_{0}\left(A_{y}\right) \sin k_{y} \Gamma_{2}+H_{\text {mass }} \\
+ & \left(M-t\left(\mathcal{J}_{0}\left(A_{x}\right) \cos k_{x}-\mathcal{J}_{0}\left(A_{y}\right) \cos k_{y}-\mathcal{J}_{0}\left(A_{z}\right) \cos k_{z}\right)\right) \Gamma_{3} \\
+ & \sum_{n \in \text { odd }, n>0} \frac{2 i \eta t^{2} \mathcal{J}_{n}\left(A_{x}\right) \mathcal{J}_{n}\left(A_{y}\right)}{n \omega} \sin \frac{n \pi}{2} \\
\times & \left(\cos k_{x} \cos k_{y}\left[\Gamma_{1}, \Gamma_{2}\right]+\cos k_{x} \sin k_{y}\left[\Gamma_{1}, \Gamma_{3}\right]\right. \\
+ & \left.\sin k_{x} \cos k_{y}\left[\Gamma_{3}, \Gamma_{2}\right]\right)+\sum_{n \in \text { odd }, n>0} \frac{2 i \eta t^{2} \mathcal{J}_{n}\left(A_{z}\right) \mathcal{J}_{n}\left(A_{y}\right)}{n \omega} \\
\times & \sin \frac{n \pi}{2} \sin k_{z} \cos k_{y}\left[\Gamma_{3}, \Gamma_{2}\right] .
\end{aligned}
$$

Figure 4 maps out the evolution of Weyl nodes as the incident angle $\psi$ varies by using this effective Hamiltonian. The result suggests that we can manipulate the postilion of Weyl nodes by tuning the propagation direction of the incident CPL.

The leading order correction term to the continuum model can be expressed as $\Delta H=-\frac{i \eta A_{x} A_{y}}{2 \omega}\left[\Gamma_{1}, \Gamma_{2}\right]-$ $\frac{i \eta A_{y} A_{z} \sqrt{(3-M)(M-1)}}{2 \omega}\left[\Gamma_{3}, \Gamma_{2}\right]$.

Conclusions. - In this study, we uncovered the emerging Floquet WSM states in the parity-time invariant higher-order NLSM under CPL by breaking TRS. The Floquet WSM states accommodate 1D off-diagonal gapless hinge Fermi arcs caused by inherited higher-order topology from the parent NLSM. Meanwhile, the Floquet WSM states also support 2D surface Fermi arcs as in conventional WSMs. We also show that the location of Weyl nodes can be tuned by adjusting the propagation direction and incident angle of CPL.

We would like to emphasize that, unlike the Floquet WSMs in periodically driven ordinary NLSMs [21-26], the higher- order Floquet WSM here has the coexisting 1D hinge and 2D

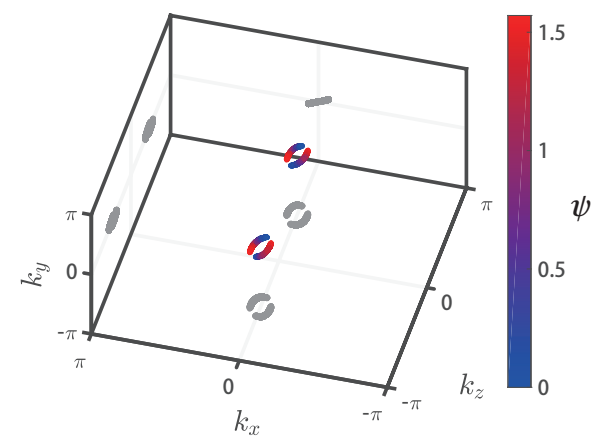

FIG. 4. The position of the light-induced Weyl points (colored dots) obtained by Eq. 10 versus the incident angle $\psi$ of CPL in the $x-z$ plane. The gray dots are the projections of the Weyl points on $k_{x}-k_{y}$, $k_{x}-k_{z}$ and $k_{y}-k_{z}$ planes, respectively. We choose model parameters the same as that used in Fig. 2.

surface Fermi arc states. Furthermore, we unveiled the dispersion of Fermi arc states can be adjusted by tuning the propagation direction of CPL. Higher-order WSMs have so far been realized only in acoustic crystals [73, 74], while our work provides a feasible way to realize tunable higher-order WSM states in non-equilibrium electronic systems. Also, it serves as a reference for future experiments on periodically driven higher-order NLSMs, such as $\mathrm{XTe}_{2}(\mathrm{X}=\mathrm{Mo}, \mathrm{W})$ [45] and 3D ABC stacked graphdiyne $[67,68,75]$.

Acknowledgments. - The authors acknowledge the support by the NSFC (under Grant Nos. 12074108, 11704106, 11974062 and 12047564), the China Postdoctoral Science Foundation (Grant No. 2019M661678), the SUSTech Presidential Postdoctoral Fellowship, the Chongqing Natural Science Foundation (Grant No. cstc2019jcyj-msxmX0563), the Fundamental Research Funds for the Central Universities of China (Grant No. 2020CDJQY-A057), and the Beijing National Laboratory for Condensed Matter Physics.
[1] Jérôme Cayssol, Balázs Dóra, Ferenc Simon, and Roderich Moessner, "Floquet topological insulators," Phys. Status Solidi RRL 7, 101 (2013).

[2] Takashi Oka and Sota Kitamura, "Floquet engineering of quantum materials," Annu. Rev. Condens. Matter Phys. 10, 387 (2019).

[3] Takashi Oka and Hideo Aoki, "Photovoltaic hall effect in graphene," Phys. Rev. B 79, 081406 (2009).

[4] Jun-ichi Inoue and Akihiro Tanaka, "Photoinduced transition between conventional and topological insulators in twodimensional electronic systems,” Phys. Rev. Lett. 105, 017401 (2010).

[5] Takuya Kitagawa, Erez Berg, Mark Rudner, and Eugene Demler, "Topological characterization of periodically driven quantum systems," Phys. Rev. B 82, 235114 (2010).

[6] Zhenghao Gu, H. A. Fertig, Daniel P. Arovas, and Assa Auerbach, "Floquet spectrum and transport through an irradiated graphene ribbon,” Phys. Rev. Lett. 107, 216601 (2011).
[7] Takuya Kitagawa, Takashi Oka, Arne Brataas, Liang Fu, and Eugene Demler, "Transport properties of nonequilibrium systems under the application of light: Photoinduced quantum hall insulators without landau levels," Phys. Rev. B 84, 235108 (2011).

[8] Pierre Delplace, Álvaro Gómez-León, and Gloria Platero, "Merging of dirac points and floquet topological transitions in ac-driven graphene," Phys. Rev. B 88, 245422 (2013).

[9] L. E. F. Foa Torres, P. M. Perez-Piskunow, C. A. Balseiro, and Gonzalo Usaj, "Multiterminal conductance of a floquet topological insulator," Phys. Rev. Lett. 113, 266801 (2014).

[10] Gonzalo Usaj, P. M. Perez-Piskunow, L. E. F. Foa Torres, and C. A. Balseiro, "Irradiated graphene as a tunable floquet topological insulator,” Phys. Rev. B 90, 115423 (2014).

[11] Netanel H Lindner, Gil Refael, and Victor Galitski, "Floquet topological insulator in semiconductor quantum wells," Nat. Phys. 7, 490 (2011).

[12] Takuya Kitagawa, Mark S. Rudner, Erez Berg, and Eugene 
Demler, "Exploring topological phases with quantum walks," Phys. Rev. A 82, 033429 (2010).

[13] Netanel H. Lindner, Doron L. Bergman, Gil Refael, and Victor Galitski, "Topological floquet spectrum in three dimensions via a two-photon resonance,” Phys. Rev. B 87, 235131 (2013).

[14] Liang Jiang, Takuya Kitagawa, Jason Alicea, A. R. Akhmerov, David Pekker, Gil Refael, J. Ignacio Cirac, Eugene Demler, Mikhail D. Lukin, and Peter Zoller, "Majorana fermions in equilibrium and in driven cold-atom quantum wires," Phys. Rev. Lett. 106, 220402 (2011).

[15] Mark S. Rudner, Netanel H. Lindner, Erez Berg, and Michael Levin, "Anomalous edge states and the bulk-edge correspondence for periodically driven two-dimensional systems," Phys. Rev. X 3, 031005 (2013).

[16] Motohiko Ezawa, "Photoinduced topological phase transition and a single dirac-cone state in silicene," Phys. Rev. Lett. 110, 026603 (2013).

[17] A. Gómez-León and G. Platero, "Floquet-bloch theory and topology in periodically driven lattices," Phys. Rev. Lett. 110, 200403 (2013).

[18] Adolfo G. Grushin, Álvaro Gómez-León, and Titus Neupert, "Floquet fractional chern insulators," Phys. Rev. Lett. 112, 156801 (2014).

[19] Rui Wang, Baigeng Wang, Rui Shen, L. Sheng, and D. Y. Xing, "Floquet weyl semimetal induced by off-resonant light," EPL 105, 17004 (2014).

[20] Hannes Hübener, Michael A Sentef, Umberto De Giovannini, Alexander F Kemper, and Angel Rubio, "Creating stable floquet-weyl semimetals by laser-driving of 3d dirac materials," Nat. Commun. 8, 1 (2017).

[21] Awadhesh Narayan, "Tunable point nodes from line-node semimetals via application of light," Phys. Rev. B 94, 041409 (2016).

[22] Zhongbo Yan and Zhong Wang, "Tunable weyl points in periodically driven nodal line semimetals," Phys. Rev. Lett. 117, 087402 (2016).

[23] Katsuhisa Taguchi, Dong-Hui Xu, Ai Yamakage, and K. T. Law, "Photovoltaic anomalous hall effect in line-node semimetals," Phys. Rev. B 94, 155206 (2016).

[24] Ching-Kit Chan, Yun-Tak Oh, Jung Hoon Han, and Patrick A. Lee, "Type-ii weyl cone transitions in driven semimetals," Phys. Rev. B 94, 121106 (2016).

[25] Rui Chen, Bin Zhou, and Dong-Hui Xu, "Floquet weyl semimetals in light-irradiated type-II and hybrid line-node semimetals," Phys. Rev. B 97, 155152 (2018).

[26] Tingwei Deng, Baobing Zheng, Fangyang Zhan, Jing Fan, Xiaozhi Wu, and Rui Wang, "Photoinduced floquet mixed-weyl semimetallic phase in a carbon allotrope," Phys. Rev. B 102, 201105 (2020).

[27] Xingfei Zhou and Guojun Jin, "Light-modulated 0- $\pi$ transition in a silicene-based josephson junction," Phys. Rev. B 94, 165436 (2016).

[28] Udit Khanna, Sumathi Rao, and Arijit Kundu, " $0-\pi$ transitions in a josephson junction of an irradiated weyl semimetal," Phys. Rev. B 95, 201115 (2017).

[29] SK Firoz Islam and A. A. Zyuzin, "Photoinduced interfacial chiral modes in threefold topological semimetal," Phys. Rev. B 100, 165302 (2019).

[30] Ankita Bhattacharya and SK Firoz Islam, "Photoinduced spin-hall resonance in a $k^{3}$-rashba spin-orbit coupled twodimensional hole system,” Phys. Rev. B 104, L081411 (2021).

[31] James W McIver, Benedikt Schulte, F-U Stein, Toru Matsuyama, Gregor Jotzu, Guido Meier, and Andrea Cavalleri, "Light-induced anomalous hall effect in graphene," Nat. Phys.
16, 38 (2020).

[32] Y. H. Wang, H. Steinberg, P. Jarillo-Herrero, and N. Gedik, "Observation of floquet-bloch states on the surface of a topological insulator,” Science 342, 453 (2013).

[33] Fahad Mahmood, Ching-Kit Chan, Zhanybek Alpichshev, Dillon Gardner, Young Lee, Patrick A Lee, and Nuh Gedik, "Selective scattering between floquet-bloch and volkov states in a topological insulator," Nat. Phys. 12, 306 (2016).

[34] Fan Zhang, C. L. Kane, and E. J. Mele, "Surface state magnetization and chiral edge states on topological insulators," Phys. Rev. Lett. 110, 046404 (2013).

[35] Wladimir A. Benalcazar, B. Andrei Bernevig, and Taylor L. Hughes, "Quantized electric multipole insulators," Science 357, 61 (2017).

[36] Josias Langbehn, Yang Peng, Luka Trifunovic, Felix von Oppen, and Piet W. Brouwer, "Reflection-symmetric second-order topological insulators and superconductors," Phys. Rev. Lett. 119, 246401 (2017).

[37] Zhida Song, Zhong Fang, and Chen Fang, " $(d-2)$-dimensional edge states of rotation symmetry protected topological states," Phys. Rev. Lett. 119, 246402 (2017).

[38] Frank Schindler, Ashley M. Cook, Maia G. Vergniory, Zhijun Wang, Stuart S. P. Parkin, B. Andrei Bernevig, and Titus Neupert, "Higher-order topological insulators," Sci. Adv. 4, eaat0346 (2018).

[39] Rui Chen, Chui-Zhen Chen, Jin-Hua Gao, Bin Zhou, and DongHui $\mathrm{Xu}$, "Higher-order topological insulators in quasicrystals," Phys. Rev. Lett. 124, 036803 (2020).

[40] Motohiko Ezawa, "Higher-order topological insulators and semimetals on the breathing Kagome and pyrochlore lattices," Phys. Rev. Lett. 120, 026801 (2018).

[41] Motohiko Ezawa, "Magnetic second-order topological insulators and semimetals," Phys. Rev. B 97, 155305 (2018).

[42] Mao Lin and Taylor L. Hughes, "Topological quadrupolar semimetals," Phys. Rev. B 98, 241103(R) (2018).

[43] Bitan Roy, "Antiunitary symmetry protected higher-order topological phases," Phys. Rev. Research 1, 032048 (2019).

[44] Benjamin J Wieder, Zhijun Wang, Jennifer Cano, Xi Dai, Leslie M Schoop, Barry Bradlyn, and B Andrei Bernevig, "Strong and fragile topological dirac semimetals with higherorder fermi arcs," Nat. Commun. 11, 1 (2020).

[45] Zhijun Wang, Benjamin J. Wieder, Jian Li, Binghai Yan, and B. Andrei Bernevig, "Higher-order topology, monopole nodal lines, and the origin of large Fermi arcs in transition metal dichalcogenides $\mathrm{XTe}_{2}(\mathrm{X}=\mathrm{Mo}, \mathrm{W}),{ }^{\prime}$ Phys. Rev. Lett. 123, 186401 (2019).

[46] Kai Wang, Jia-Xiao Dai, L. B. Shao, Shengyuan A. Yang, and Y. X. Zhao, "Boundary criticality of $\mathcal{P} \mathcal{T}$-invariant topology and second-order nodal-line semimetals," Phys. Rev. Lett. 125, 126403 (2020).

[47] Hai-Xiao Wang, Zhi-Kang Lin, Bin Jiang, Guang-Yu Guo, and Jian-Hua Jiang, "Higher-order weyl semimetals," Phys. Rev. Lett. 125, 146401 (2020).

[48] Sayed Ali Akbar Ghorashi, Tianhe Li, and Taylor L. Hughes, "Higher-order weyl semimetals," Phys. Rev. Lett. 125, 266804 (2020).

[49] Y. X. Zhao, Cong Chen, Xian-Lei Sheng, and Shengyuan A. Yang, "Switching spinless and spinful topological phases with projective PT symmetry," Phys. Rev. Lett. 126, 196402 (2021).

[50] W. B. Rui, Zhen Zheng, Moritz M. Hirschmann, Song-Bo Zhang, Chenjie Wang, and Z. D. Wang, "Intertwined weyl phases: higher-order topology meets unconventional weyl fermions via crystalline symmetry," arXiv:2105.08443.

[51] Raditya Weda Bomantara, Longwen Zhou, Jiaxin Pan, and 
Jiangbin Gong, "Coupled-wire construction of static and floquet second-order topological insulators," Phys. Rev. B 99, 045441 (2019).

[52] Martin Rodriguez-Vega, Abhishek Kumar, and Babak Seradjeh, "Higher-order floquet topological phases with corner and bulk bound states," Phys. Rev. B 100, 085138 (2019).

[53] Tanay Nag, Vladimir Juričić, and Bitan Roy, "Out of equilibrium higher-order topological insulator: Floquet engineering and quench dynamics," Phys. Rev. Research 1, 032045 (2019).

[54] Ranjani Seshadri, Anirban Dutta, and Diptiman Sen, "Generating a second-order topological insulator with multiple corner states by periodic driving," Phys. Rev. B 100, 115403 (2019).

[55] Yang Peng and Gil Refael, "Floquet second-order topological insulators from nonsymmorphic space-time symmetries," Phys. Rev. Lett. 123, 016806 (2019).

[56] Haiping Hu, Biao Huang, Erhai Zhao, and W. Vincent Liu, "Dynamical singularities of floquet higher-order topological insulators," Phys. Rev. Lett. 124, 057001 (2020).

[57] Arnob Kumar Ghosh, Ganesh C. Paul, and Arijit Saha, "Higher order topological insulator via periodic driving," Phys. Rev. B 101, 235403 (2020).

[58] Yang Peng, "Floquet higher-order topological insulators and superconductors with space-time symmetries," Phys. Rev. Research 2, 013124 (2020).

[59] Raditya Weda Bomantara, "Time-induced second-order topological superconductors," Phys. Rev. Research 2, 033495 (2020).

[60] Biao Huang and W. Vincent Liu, "Floquet higher-order topological insulators with anomalous dynamical polarization," Phys. Rev. Lett. 124, 216601 (2020).

[61] Weiwei Zhu, Y. D. Chong, and Jiangbin Gong, "Floquet higherorder topological insulator in a periodically driven bipartite lattice,” Phys. Rev. B 103, L041402 (2021).

[62] Rui-Xing Zhang and Zhi-Cheng Yang, "Tunable fragile topology in floquet systems," Phys. Rev. B 103, L121115 (2021).

[63] Tanay Nag, Vladimir Juričić, and Bitan Roy, "Hierarchy of higher-order floquet topological phases in three dimensions," Phys. Rev. B 103, 115308 (2021).

[64] Weiwei Zhu, Y. D. Chong, and Jiangbin Gong, "Symmetry analysis of anomalous floquet topological phases," Phys. Rev. B 104, L020302 (2021).

[65] Weiwei Zhu, Muhammad Umer, and Jiangbin Gong, "Floquet higher-order weyl and nexus semimetals," Phys. Rev. Research 3, L032026 (2021).

[66] W. B. Rui, Song-Bo Zhang, Moritz M. Hirschmann, Zhen Zheng, Andreas P. Schnyder, Björn Trauzettel, and Z. D. Wang, "Higher-order weyl superconductors with anisotropic weyl-point connectivity,” Phys. Rev. B 103, 184510 (2021).

[67] Junyeong Ahn, Dongwook Kim, Youngkuk Kim, and BohmJung Yang, "Band topology and linking structure of nodal line semimetals with $Z_{2}$ monopole charges," Phys. Rev. Lett. 121, 106403 (2018).

[68] Eunwoo Lee, Rokyeon Kim, Junyeong Ahn, and Bohm-Jung Yang, "Two-dimensional higher-order topology in monolayer graphdiyne," npj Quantum Mater. 5, 1 (2020).

[69] Jon H. Shirley, "Solution of the schrödinger equation with a hamiltonian periodic in time," Phys. Rev. 138, B979 (1965).

[70] Hideo Sambe, "Steady states and quasienergies of a quantummechanical system in an oscillating field," Phys. Rev. A 7, 2203 (1973).

[71] Marin Bukov, Luca D'Alessio, and Anatoli Polkovnikov, "Universal high-frequency behavior of periodically driven systems: from dynamical stabilization to floquet engineering," Adv. Phys. 64, 139 (2015).

[72] André Eckardt and Egidijus Anisimovas, "High-frequency approximation for periodically driven quantum systems from a floquet-space perspective,” New J. Phys. 17, 093039 (2015).

[73] Li Luo, Hai-Xiao Wang, Zhi-Kang Lin, Bin Jiang, Ying Wu, Feng Li, and Jian-Hua Jiang, "Observation of a phononic higher-order weyl semimetal,” Nat. Mater. 20, 794 (2021).

[74] Qiang Wei, Xuewei Zhang, Weiyin Deng, Jiuyang Lu, Xueqin Huang, Mou Yan, Gang Chen, Zhengyou Liu, and Suotang Jia, "Higher-order topological semimetal in acoustic crystals," Nat. Mater. 20, 812 (2021).

[75] Cong Chen, Xu-Tao Zeng, Ziyu Chen, Y. X. Zhao, Xian-Lei Sheng, and Shengyuan A. Yang, "Second-order real nodal-line semimetal in three-dimensional graphdiyne," Phys. Rev. Lett. 128, 026405 (2022). 